\title{
Interneuronal Basis of the Generation of Related but Distinct Motor Programs in Aplysia: Implications for Current Neuronal Models of Vertebrate Intralimb Coordination
}

\author{
Jian Jing and Klaudiusz R. Weiss \\ Department of Physiology and Biophysics, Mount Sinai School of Medicine, New York, New York 10029-6574
}

Coordination of two sets of movements, protraction-retraction versus opening-closing, of the feeding apparatus (the radula) in ingestive and egestive motor programs of Aplysia resembles vertebrate intralimb coordination in that the relative timing of the two sets of movements differs in the two motor programs. In both ingestion and egestion, radula protraction and retraction alternate, whereas radula closure shifts its phase relative to protraction-retraction. In egestion, the radula closes in protraction; in ingestion, the radula closes in retraction. In both ingestive and egestive motor programs elicited by the command-like neuron, cerebral-buccal interneuron-2 (CBI-2), the protraction and retraction movements are mediated by the same sets of controller interneurons. In contrast, radula closure is mediated by two controller interneurons, B20 and B40, that are preferentially active in egestion and ingestion, respectively. In egestion, B20, active in protraction, drives closure motorneuron B8 in

Complex motor acts often involve coordinated movements of multiple sets of agonist and antagonist muscles (Grillner, 1981, 1985; Stein and Smith, 1997; Orlovsky et al., 1999). Animals can produce multiple, related behaviors, e.g., different forms of locomotion, rostral, pocket, and caudal scratch, ingestion, and egestion (Kupfermann, 1974; Grillner, 1981; Mortin et al., 1985; Buford et al., 1990; Grasso et al., 1998), by generating distinct coordinated motor patterns, referred to as motor programs (Ayers and Davis, 1977) or forms of a motor task (Mortin et al., 1985). One common strategy of generating distinct motor programs consists of changing the relative timing of different sets of movements (Mortin et al., 1985; Smith et al., 1985, 1998a; Chrachri and Clarac, 1990; Morton and Chiel, 1993a; Combes et al., 1999).

In principle, different forms of coordination of multiple sets of movements could be implemented in a variety of ways. Two hypothetical models for intralimb coordination in spinal circuits (Stein and Smith, 1997) were proposed. Grillner (1981, 1985) proposed that agonist muscles (e.g., around one joint) are activated by a controller, termed a unit burst generator, or a module

Received Feb. 12, 2002; revised April 23, 2002; accepted April 23, 2002.

This research was supported by National Institute of Mental Health Grants RO1 MH50235, KO5 MH 01427, and F32 MH12890. The National Resource for Aplysia of the University of Miami provided some of the animals used in this study under Grant RR-10294 from the National Center for Research Resources, National Institutes of Health. We thank Dr. E. C. Cropper for comments on an earlier version of this manuscript.

Correspondence should be addressed to Dr. Jian Jing, Department of Physiology and Biophysics, Box 1218, Mt. Sinai School of Medicine, 1 Gustave Levy Place, New York, NY 10029. E-mail: jjing@inka.mssm.edu.

Copyright (C) 2002 Society for Neuroscience $0270-6474 / 02 / 226228-11 \$ 15.00 / 0$ protraction, whereas in ingestion, B40, also active in protraction, uses a functionally novel mechanism, fast inhibition and slow excitation, to drive B8 in retraction. Our findings are summarized in a neural model that permits a conceptual comparison of our model with two previous hypothetical models of intralimb coordination in spinal circuits that were proposed by Grillner (1981, 1985) and Berkowitz and Stein (1994). Although our model supports the existence of separate controllers for different movements as in the Grillner $(1981,1985)$ model; in terms of basic mechanisms, our model is similar to the Berkowitz and Stein (1994) model because the closure movement is mediated by separate controllers in different programs, and thus both models can be classified as recruitment models.

Key words: Aplysia; feeding; ingestion; egestion; central pattern generator; mollusc; motor program; motor pattern selection; interneuron; intralimb coordination
(Jordan, 1991; Stein et al., 1995). Coordination of sets of joint movements in different behaviors can be achieved by switching the sign of the phase coupling between the controllers of different joints of the limb through descending inputs. Grillner's (1981, 1985) hypothesis has been used to explain multiple forms of cat walking (Smith et al., 1998a,b). The second model (Berkowitz and Stein, 1994) was proposed to explain the observation that during a rostral scratch in turtle, knee extension occurs during hip flexion, whereas during pocket scratch, knee extension occurs during hip extension (Mortin et al., 1985; Robertson et al., 1985). The model states that in rostral scratch, the knee extensor is activated by the controllers for hip flexor, whereas in pocket scratch, the knee extensor is activated by controllers for hip extensor.

Because of the large number of interneurons involved, these models are difficult to verify in spinal circuits. The Aplysia CNS is amenable to testing of specific models of coordination (Jing and Weiss, 2001). In Aplysia, two forms of feeding, ingestion and egestion, involve different coordination patterns of two sets of movements of the feeding apparatus (radula), protraction and retraction versus opening and closing. In both motor programs, when Aplysia transitions from a quiescent to an active state, protraction occurs first and is followed by retraction. However, in ingestion, the radula closes during retraction to pull food in; in egestion, the radula closes during protraction to push inedible objects out (Morton and Chiel, 1993a). Thus, we consider the protraction-retraction movements to be phase-fixed movements (PFMs), and we consider the radula closure movement to be a phase-shifting movement (PSM). In ingestion and egestion, the same sets of interneurons mediate the PFMs (Jing et al., 1999; 
Jing and Weiss, 2001). We now show that the PSM in ingestion is mediated by a separate interneuron from the interneuron that mediates the PSM in egestion (Jing and Weiss, 2001). Comparison with the Grillner (1981, 1985) and Berkowitz and Stein (1994) models suggests that Aplysia circuits display both similarities and differences with the two spinal models.

\section{MATERIALS AND METHODS}

Specimens of Aplysia californica were obtained from Marinus (Long Beach, CA) and from the National Resource for Aplysia at the University of Miami. They were maintained in circulating artificial seawater (ASW), made from Instant Ocean (Aquarium Systems, Mentor, $\mathrm{OH}$ ) at $14-15^{\circ} \mathrm{C}$. Animals weighing 50-250 gm were anesthetized by injection ( $50 \%$ of the body weight) of isotonic $\mathrm{MgCl}_{2}(337 \mathrm{mM})$. Cerebral ganglia together with buccal ganglia were dissected out and desheathed. The ganglia were then pinned in a chamber that had a volume of $\sim 1.5 \mathrm{ml}$. The preparation was continuously perfused with ASW (in mM: $460 \mathrm{NaCl}, 10 \mathrm{KCl}, 55 \mathrm{MgCl}_{2}$, $11 \mathrm{CaCl}_{2}$, and $10 \mathrm{HEPES}$ buffer), $\mathrm{pH} 7.6$, at a rate of $0.3 \mathrm{ml} / \mathrm{min}$, and maintained at $14-17^{\circ} \mathrm{C}$. All chemicals were purchased from Sigma (St. Louis, MO).

Standard intracellular and extracellular recordings were obtained as described (Jing and Weiss, 2001). Digitized data were plotted with Axum (Mathsoft, Cambridge, MA). Functional synaptic connections were examined in normal saline, and the ability of postsynaptic potentials (PSPs) to follow presynaptic spikes one-for-one was taken as an indication of probable monosynapticity. Assays of monosynapticity were conducted in high-divalent saline (in mM: $312 \mathrm{NaCl}, 10 \mathrm{KCl}, 132 \mathrm{MgCl}_{2}, 33 \mathrm{CaCl}_{2}$, and 10 HEPES), $\mathrm{pH} 7.6$, a solution that elevates spike thresholds and thus curtails polysynaptic activation. Neurons were identified based on location, size, electrophysiological, and morphological characteristics (Jing and Weiss, 2001).

Throughout the results, $n$ refers to the number of preparations, unless otherwise stated.

\section{RESULTS}

\section{Differential activity of B40 and B20 in ingestion and egestion}

Ingestive and egestive behaviors of Aplysia were initially observed in behaving animals (Kupfermann, 1974). The specific timing of the two sets of radula movements, protraction and retraction versus opening and closing, involved in these behaviors has been analyzed in intact animals with video recording (Morton and Chiel, 1993a) that showed that in ingestion, the radula closes during retraction; whereas in egestion, the radula closes during protraction. These observations have been verified in reduced preparations in which muscles or motor nerves that mediate the radula movements have been recorded (Cropper et al., 1990; Morton and Chiel, 1993b). Numerous other studies have shown that these timing differences observed in actual behaviors can also be extended to neural activity observed in fictive ingestive and egestive motor programs in the isolated CNS (Susswein and Byrne, 1988; Church and Lloyd, 1994; Hurwitz et al., 1997; Nargeot et al., 1997; Kabotyanski et al., 1998; Sanchez and Kirk, 2000; Jing and Weiss, 2001; Morgan et al., 2002). In the present study, we elicited ingestive and egestive motor programs through stimulation of the cerebral-buccal interneuron-2 (CBI-2; Rosen et al., 1991). CBI-2 is a command-like interneuron that is activated by appropriate sensory stimuli. Furthermore, when CBI-2 is activated through current injection, it can elicit both ingestive and egestive motor programs (Rosen et al., 1991; Church and Lloyd, 1994; Jing and Weiss, 2001; Morgan et al., 2002). In a given preparation, however, if the stimulation paradigm remains the same, the motor programs elicited by stimulation of CBI- 2 stabilize after several episodes of CBI-2 stimulation, and the type of motor programs elicited by stimulation of CBI-2 alone remains the same thereafter throughout the experiment.
The radula protraction, retraction, and closing movements are mediated by feeding interneurons located in the buccal ganglion. Previous data indicate that the buccal interneurons, which control protraction (B34, B63) (Hurwitz et al., 1997) and retraction (B64) (Hurwitz and Susswein, 1996) are active in both ingestive and egestive motor programs (Jing et al., 1999; Jing and Weiss, 2001). Furthermore, the interneuron B20, active in protraction, is the major driver of radula closure motorneuron B8 in egestive motor programs (Jing and Weiss, 2001). An interneuron that controls B8 in ingestive motor programs, however, has not been identified. Because B8 is active in the retraction phase, we hypothesized that the interneuron that controls radula closure in ingestion may exhibit the following properties: (1) the interneuron should be preferentially active in ingestive rather than egestive motor programs, (2) this neuron should be active in the retraction phase, and (3) this neuron should excite directly radula closure motor neurons through fast excitation. Our extensive search for an interneuron that meets all of the above criteria was unsuccessful. We did, however, find a neuron, which we named B40, that plays a major role in ingestive motor programs, but is active in protraction, rather than retraction, of the motor programs. B40 is located on the caudal surface of the buccal ganglion, posteriorly to B34, and is a bilaterally symmetrical cell. Similar to B63 and B34, B40 is a buccal-cerebral interneuron that sends its sole axon out of the buccal ganglion to the cerebral ganglion through the contralateral cerebral-buccal connective (CBC).

In the feeding motor programs elicited by CBI-2 (Figs. 1, 2), protraction is monitored by activity in the $\mathrm{I} 2$ nerve, which contains the axons of protraction motor neurons B31/32 and B61/62 (Hurwitz et al., 1996). Retraction, mediated by the retractionphase interneuron B64, is monitored by periods of hyperpolarization in either B40 or B20 (Jing and Weiss, 2001) after the end of protraction. The types of motor programs can be determined based on the activity pattern of the radula closure motor neuron B8. In ingestive motor programs, B8 is weakly active in protraction and strongly active in retraction, whereas in egestive motor programs, B8 is strongly active in protraction and is not active or is weakly active in retraction (Fig. 1, compare $A, B$ ). Based on the quantitative study of B8 activity patterns in a large number of motor programs (compare Fig. $1 C$ ), $\mathrm{CBI}-2$ was recently found to produce a third type of motor programs, in which $\mathrm{B} 8$ fires at similar rates in protraction and retraction, and which was named the intermediate (ambiguous) program (Morgan et al., 2002).

Like interneurons B63, B34, and B20, B40 is active in the protraction phase of motor programs, but unlike these interneurons, it is preferentially active in ingestive motor programs (Fig. $1 A, C 1)$. We have shown that $\mathrm{B} 20$ is more active in egestion than in ingestion (Jing and Weiss, 2001), thus, B40 activity level is opposite to that of B20 in the two types of motor programs (Fig. $1 B, C 2)$. To further examine the causal relationship between activity levels of B40 and B20 and the types of motor programs, we quantified the average firing rate of B8 during protraction and retraction as well as the average firing rate of B40 and B20 in different types of motor programs. These motor programs were elicited by stimulation of CBI- 2 with DC or current pulses and are from 47 episodes recorded from 32 preparations. The data are plotted in three-dimensional (3-D) graphs (Fig. 1C). In the graphs, the $x-y$ axis is B8 firing frequency in protraction and retraction, and the $z$-axis is B40 or B20 firing frequency. Based on a 2-D plot of B8 activity in protraction and retraction in different motor programs, Morgan et al. (2002) have used cluster analysis to classify these motor programs into three groups: ingestive (low 


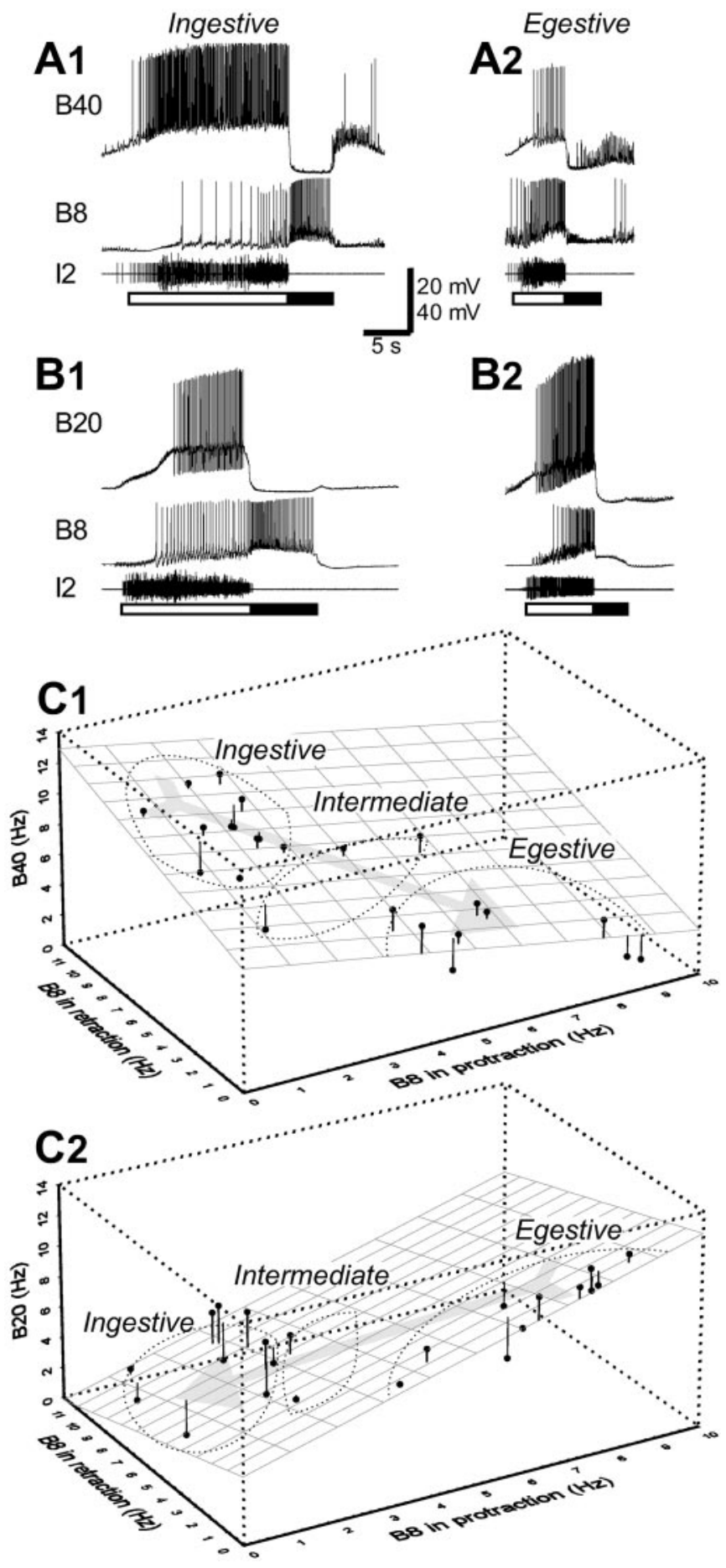

Figure 1. B40 is preferentially active in ingestion, whereas $\mathrm{B} 20$ is preferentially active in egestion. $A, B$, Examples showing $\mathrm{B} 40(A)$ and $\mathrm{B} 20(B)$ activity in ingestive (left panels) and egestive (right panels) motor programs elicited by CBI-2. Protraction (open bar) is monitored by activity in the I2 nerve, which contains the axons of protraction motor neurons. Retraction (filled bar) is monitored by periods of hyperpolarization in either B40 or B20 after the end of protraction, which is mediated by the retraction-phase interneuron B64 (compare with Fig. $8 C$ ). In the left panels, B8 fires weakly in protraction and strongly in retraction, thus the programs are ingestive. In the right panels, B8 only fired during protraction, not in retraction, thus the programs are egestive. B40 is more active in ingestion than egestion, whereas $\mathrm{B} 20$ is more active in egestion than ingestion. $C$, 3-D regression plots of B40 and B20 activity in relation to B8 activity in the protraction and retraction phases of motor programs. In a total of 47
B8 firing in protraction and high B8 firing in retraction), egestive (high B8 firing in protraction and low B8 firing in retraction), intermediate (similar B8 firing in protraction and retraction). Similarly, the data points in the 3-D plots can also be grouped into three clusters along the $x-y$ axis plane. Specifically, along the $x-y$ axis plane, the data points that are located at top-left corner are ingestive, whereas the data points that are located at the bottomright corner are egestive. Three-dimensional regression planes, generated using ordinary least-squares (OLS) to calculate $Z$ values for a given $X$ and $Y$, are drawn to show the relationship of B40/B20 activity with different types of motor programs. Most data points fell in the regression planes (shown as only the dots) or close by (shown as the dots with a vertical line that joins the dot with the regression plane, the shorter the line, the better the fit), suggesting the regression provides a good fit. For B40, when the data points are in the top-left corner (ingestive), B40 activity is the highest $(\sim 11 \mathrm{~Hz})$, and as the programs shift from top-left corner toward the bottom-right corner (egestion), B40 activity drops (the regression plane declines). In contrast, B20 activity is the highest $(\sim 11 \mathrm{~Hz})$ when the data points are clustered in bottom-right corner (egestive) and as the programs shift from the bottom-right corner toward the top-left corner (ingestion), B20 activity drops precipitously (the regression plane declines). Finally, we calculated the average frequency of B40/B20 in the three groups. Average firing frequency ( \pm SEM) of B40 in ingestive motor programs is $9.85 \pm 0.33(n=12)$, in intermediate programs is $7.93 \pm 0.57(n=3)$, and in egestive programs is $5.32 \pm 0.73(n=9)$. Average firing frequency of B20 in egestive motor programs is $9.41 \pm 0.61(n=11)$, in intermediate programs is $5.21 \pm 0.53(n=2)$, and in ingestive programs is $2.89 \pm 0.75$ $(n=10)$. Thus, these data provide strong supporting evidence that B40 and B20 may be important controllers of radula closure motor neuron B8 for ingestive and egestive motor programs, respectively.

\section{Functional role of B40 in ingestive motor programs}

In the earlier report (Jing and Weiss, 2001), we showed that B20s are necessary for strong B8 firing during protraction in egestive motor programs by hyperpolarizing bilateral B20s. In the second series of the current experiments, we performed similar experiments on B40 to determine if B40 is indeed an important controller of B8 phasing in ingestion. Ingestive motor programs elicited by CBI-2 were monitored by activity in the radula closer B8 (which fired weakly in protraction and fired strongly in retrac-

motor programs, B8 activity in protraction and retraction as well as B40 or B20 activity are quantified and plotted in the 3-D graphs. The data can be grouped into three clusters (encircled by dotted lines) along the $x-y$ axis plane. In the top left group, B8 is more active in retraction than in protraction, and these programs are ingestive; in the bottom right group, B8 is more active in protraction than in retraction, and these programs are egestive; in the middle group, B8 fires similarly in protraction and retraction, and thus these programs are intermediate (Morgan et al., 2002). 3-D regression planes are drawn and the data points fall within the plane (shown only as the dots) or close by (shown as the dots with a vertical line that joins the dot with the regression plane; the shorter the line, the better the fit), suggesting the regression provides a good fit. Along the $x-y$-axis plane, B40 activity is highest in the top left corner (ingestive), and when data points shift from top left (ingestive) to bottom right (egestive) direction ( gray arrow), B40 activity drops, indicating that B40 is more active in ingestion. Conversely, B20 activity is highest in the bottom right corner (egestive) and when data points shift from bottom right (egestive) to top left (ingestive) direction (gray arrow), B20 activity drops, indicating that $\mathrm{B} 20$ is more active in egestion. 

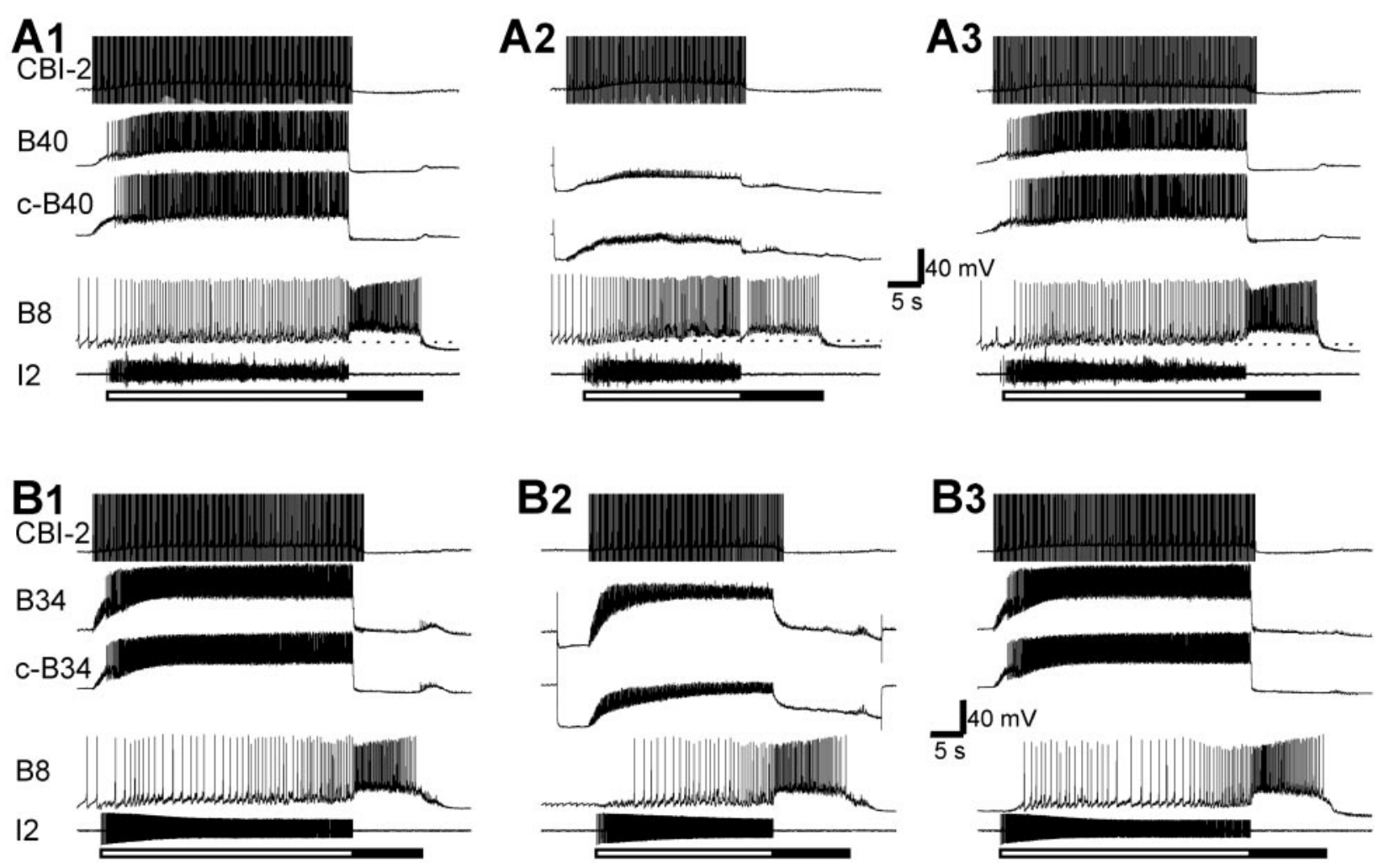

Figure 2. B40 is critical for expression of ingestive activity patterns of radula closure motor neuron B8. Motor programs were elicited by stimulation of CBI-2 with brief current pulses. The three panels in $A$ and $B$ represent two series of three episodes of CBI- 2 stimulation ( 2 min apart) in two different preparations. $A$, Stimulation of CBI-2 alone consistently elicited ingestive programs $(A 1, A 3)$ with strong activity in the ipsilateral B40 and the contralateral B40 (c-B40), because B8 fired high-frequency bursts during retraction ( filled bar), which follows protraction (I2, open bar). Hyperpolarization of both $\mathrm{B} 40 \mathrm{~s}$ reduced activity of $\mathrm{B} 8$ during retraction and increased activity of $\mathrm{B} 8$ during protraction, i.e., made the program more egestive ( $A 2$ ). Dotted lines in $B 8$ traces show resting membrane potentials. $B$, Both the ipsilateral B34 and the contralateral B34 (c-B34) were strongly active in CBI-2-elicited ingestive programs $(B 1, B 3)$, but hyperpolarization of both B34s had little impact on activity pattern of B8 $(B 2)$.

tion). B40s fired at high frequency during protraction (Fig. 2A1). However, when B40s were bilaterally hyperpolarized, B8 fired more during protraction and fired less during retraction (Fig. $2 A 2$ ), and the program became intermediate rather than ingestive (compare Fig. 3). B8 activity returned to the ingestive pattern when B40s were not hyperpolarized in the third motor program elicited by CBI-2 (Fig. 2A3).

To determine whether the effect of B40 on the B8 firing pattern in ingestive motor programs is specific, we also performed similar experiments on another protraction-phase interneuron, B34. We have shown earlier (Jing and Weiss, 2001) that B34s are similarly active in both ingestion and egestion elicited by CBI-2, and in another study (Hurwitz et al., 1997), B34 has been shown to provide strong synaptic excitation to motor neurons that produce radula protraction movements. The evidence suggests that B34 is an important mediator for protraction movements. Bilateral hyperpolarization of B34s in ingestive motor programs had a minimum impact on B8 firing pattern in ingestion (Fig. 2B). This indicates that B34 is not an important controller element of radula closure for ingestion, consistent with the earlier study (Jing and Weiss, 2001).

The effects of B40 $(n=5)$ and B34 $(n=3)$ hyperpolarization on $\mathrm{B} 8$ firing pattern in CBI-2 elicited ingestive motor programs are summarized in a plot shown in Figure 3 ( $x$-axis, B8 firing frequency in protraction vs $y$-axis, B8 firing frequency in retraction). In the plot, each experiment is represented by two data points connected by a line (dashed lines for B40, straight lines for B34). For each pair of data points, the top one represents the data obtained before hyperpolarization of B40 or B34, and the bottom one represents data obtained during hyperpolarization of B40 or B34. Dotted lines encircling the data points show three distinct clusters derived from cluster analysis (Morgan et al., 2002) that represent ingestive, intermediate, and egestive motor programs (compare Fig. 1C). Thus, all the motor programs were ingestive before hyperpolarization of B40 or B34. Hyperpolarization of B40s shifted B8 activity patterns from top-left corner to bottomright direction, i.e., from ingestive programs to intermediate programs. In other words, B8 now fired at similar rates in both protraction and retraction, which is characteristic of intermediate programs. Note that all B40 hyperpolarization experiments were conducted in preparations in which CBI-2 stimulation consistently elicited ingestive motor programs, because the purpose of these experiments was to examine the functional roles of B40 in ingestion. Overall, in this study (47 episodes in 32 preparations), we found that under our conditions of CBI-2 stimulation, without B40 hyperpolarization, $46.8 \%$ of motor patterns elicited by CBI-2 stimulation were ingestive, $42.6 \%$ of motor patterns were egestive, and $10.6 \%$ of motor patterns were intermediate. Although the B8 firing was altered somewhat when B34s were hyperpolarized, the motor programs remained ingestive. Thus, B40, but not $\mathrm{B} 34$, is critical for expression of ingestive motor programs elicited by CBI-2. 


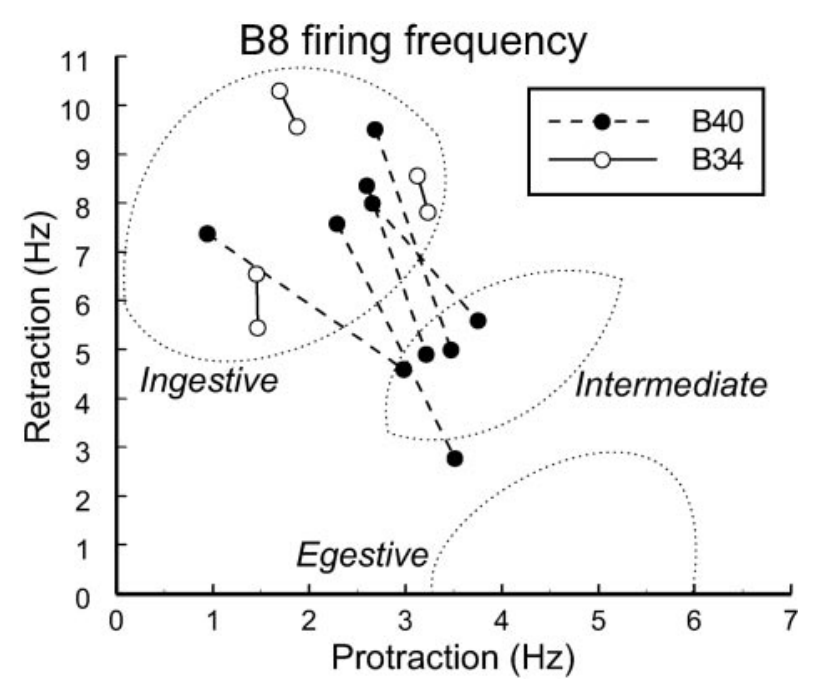

Figure 3. Plot of group data showing the effect of hyperpolarization of both $\mathrm{B} 40$ s or $\mathrm{B} 34 \mathrm{~s}$ on radula closure (B8) firing frequency in CBI-2elicited motor programs. For each experiment, there is a pair of data points that are connected by a line. For each pair, the data points in the top part of the graph were obtained in the absence of hyperpolarization of B40 or B34. The other data points were obtained during hyperpolarization of B40 or B34. Dotted lines encircling the data points show the three clusters derived from cluster analysis (Morgan et al., 2002) that categorized motor programs into ingestive, intermediate, and egestive programs. All the initial programs were ingestive as B8 fired at a low frequency in protraction and at a high frequency in retraction. When the B40s were hyperpolarized, motor programs became more egestive, i.e., B8 activity shifted toward protraction, indicating that B40 is a critical element for expression of CBI-2-elicited ingestive motor programs. Hyperpolarization of B34s also shifted B8 activity somewhat, but the motor programs remained ingestive. Thus, in contrast to B40, B34 is not critical for the expression of ingestive motor programs.

\section{Control of the radula closure motoneuron B8 by the interneuron B40}

To examine how B40 controls the activity of the radula closure B8, we studied the synaptic connection from B40 to B8. The PSPs that B40 elicited in B8 had dual components, fast IPSPs following presynaptic spikes one-for-one and slow EPSPs occurring at a delay from the onset of B40 activity $(n=27)$ (Fig. 4). As shown in Figures 2 and 3, hyperpolarization of B40 causes more B8 firing in protraction and less B8 firing in retraction, indicating that the normal function of $\mathrm{B} 40$ is to reduce $\mathrm{B} 8$ firing in protraction when B40 is active and promote B8 firing in retraction when B40 ceases firing. The dual function can be efficiently accomplished by the dual-component PSPs. Because the fast IPSPs followed B40 presynaptic spikes one for one (Fig. 4A2, B2), and both the fast IPSPs and slow EPSPs persisted in high-divalent saline $(n=18)$ (Fig. $4 B, C)$, both components of the PSPs from B40 to B8 are monosynaptic. The slow EPSPs were not obvious when B40 was stimulated for a brief time $(\leq 250 \mathrm{msec}$ ) (Fig. $4 C 1, C 2$ ) and began to appear when B40 was stimulated for $>500$ msec (Fig. 4C3-6). This suggested that slow EPSPs may not follow B40 spikes one-for-one and may require multiple B40 spikes. We also examined if postinhibitory rebound of B8 may contribute to the slow depolarization that is most prominent when B40 ceases firing because during B40 stimulation, B8 is hyperpolarized by the fast IPSPs. Toward this goal, we delivered hyperpolarizing current pulses of the same duration and similar amplitude with the hyperpolarizations caused by B40 stimulation, and we found that the postinhibitory rebound was too small to account for the slow depolarization (Fig. 4C4-C6). This experiment indicates that the slow depolarization represents slow synaptic potential that is elicited by B40.

To further characterize the properties of the PSPs that B40 elicits in B8, we examined whether the PSPs are associated with conductance change in $\mathrm{B} 8(n=3)$ and whether they are dependent on the membrane potentials of $\mathrm{B} 8(n=4)$. We measured the B8 conductance by applying a small hyperpolarizing current pulse $(<0.5 \mathrm{nA})$ of $500 \mathrm{msec}$ duration at $0.5 \mathrm{~Hz}$ (Fig. $5 A$ ). During B40 stimulation when the fast IPSPs were most prominent, the voltage deflections in B8 caused by the current pulses became smaller indicating that the fast IPSPs are associated with conductance increase. On the other hand, immediately after B40 stimulation when the slow EPSPs were most prominent, the voltage deflections in B8 caused by current pulses became larger indicating that the slow EPSPs are associated with conductance decrease. These conductance changes in B8 elicited by B40 are present in both normal saline (Fig. 5A1) and high-divalent saline (Fig. 5A2). To test whether $\mathrm{B} 8$ membrane potentials affect the PSPs elicited by B40, we stimulated B40 with brief current pulses for $1 \mathrm{sec}$ at $20 \mathrm{~Hz}$ (Fig. 5B) and changed B8 membrane potential by current injection through a second electrode. The fast IPSPs reversed to become depolarizing potentials when $\mathrm{B} 8$ membrane potentials were held below $-80 \mathrm{mV}$. However, the slow EPSPs became smaller when $\mathrm{B} 8$ was more hyperpolarized and did not reverse even when $\mathrm{B} 8$ membrane potentials were held at $-90 \mathrm{mV}$.

Previously, it was reported that B34 also elicited fast IPSPs and slow EPSPs in B8 (Hurwitz et al., 1997). The slow EPSPs were also found to be associated with conductance decrease similar to those elicited by B40. Slow synaptic potentials that are associated with conductance decrease and become smaller when the postsynaptic cell is hyperpolarized have also been demonstrated in other parts of Aplysia feeding network (Chiel et al., 1986; Weiss et al., 1986).

Because hyperpolarization of B40s had a significant impact on B8 activity pattern in ingestive motor programs, i.e., B8 fired more in protraction and fired less in retraction (Figs. 2, 3), we examined the effect of B40 activity on B8 excitability (Fig. $6 A$ ) to determine whether the fast IPSPs and slow EPSPs were responsible for the B40 functional role. In addition, hyperpolarization of B34s had a small effect on B8 activity pattern in ingestive programs, although B34 also elicited fast IPSPs and slow EPSPs in B8. Therefore, we also examined the effects of B34 on B8 excitability (Fig. $6 B$ ) and compared these effects with those of B40 (Fig. 7). In these experiments (Figs. 6, 7), B8 excitability was tested by $3 \mathrm{sec}$ constant current pulses every $30 \mathrm{sec}$ and number of B8 spikes during the current pulses was counted. For the experiments to be comparable with each other, in the beginning of each experiment, the size of current pulses was adjusted so that B8 fired $\sim 12$ spikes and remained the same throughout the rest of the experiment.

Two stimulation paradigms were used to examine the effect of B40/B34 activity on B8 excitability. In the first stimulation paradigm, B40 (Figs. $6 A 1,7 A 1$ ) or B34 (Figs. $6 B 1,7 B 1$ ) was stimulated $300 \mathrm{msec}$ before and throughout the current pulses injected into B8. This paradigm examines functional effects of the fast IPSPs and simulates the situation in a feeding motor program, where B40/B34 fire together with B8 during protraction. Under such conditions, B40 activity reduced B8 excitability (Fig. 6A1), whereas B34 activity slightly increased B8 excitability (Fig. 6B1). Group data are shown in Figure 7, $A 1$ (B40) and B1 (B34). For B40 effect $(n=11)$, one-way repeated measures ANOVA on the 


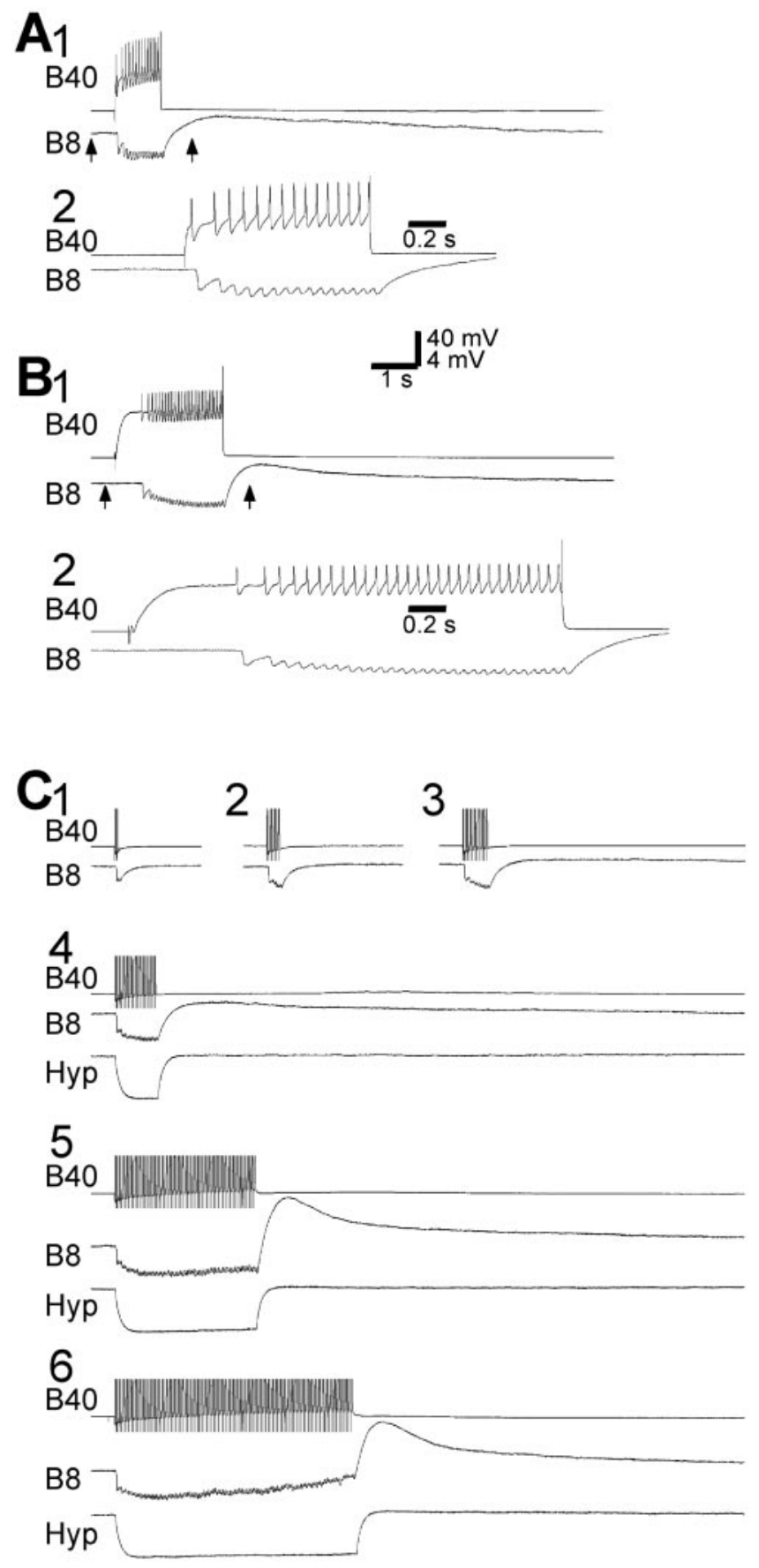

Figure 4. B40 elicits fast inhibitory and slow excitatory responses in the contralateral B8. $A, B$, The fast IPSPs and slow EPSPs that B40 elicited in B8 were present in both normal saline $(A)$ and high-divalent saline $(B)$. $A 2$ and $B 2$ are expanded records of $A 1$ and $B 1$ (between arrows), respectively, which were plotted to show more clearly that the fast IPSPs followed B40 presynaptic spikes one-for-one. $C$, B40 was stimulated with $20 \mathrm{~Hz}$ trains of brief current pulses for different periods of time. The slow EPSPs became obvious only when B40 was stimulated for $>500 \mathrm{msec}$. The durations of B40 stimulation were $100 \mathrm{msec}$ (2 spikes, $C 1), 250 \mathrm{msec}(C 2)$, $500 \mathrm{msec}(C 3), 1 \mathrm{sec}(C 4), 3 \mathrm{sec}(C 5)$, or $5 \mathrm{sec}(C 6)$. No slow EPSPs were obvious when B40 stimulation was $<250 \mathrm{msec}(C 1, C 2)$. The slow EPSPs began to appear when B40 stimulation lasted for $\geq 500 \mathrm{msec}$. To examine if postinhibitory rebound may contribute to the slow EPSPs, hyperpolarizing (Hyp) current pulses of similar size and duration as those caused by B40 stimulation were applied in B8 for $1 \mathrm{sec}(C 4), 3 \mathrm{sec}(C 5)$, or $5 \mathrm{sec}$ (C6) and were plotted as the last trace in the three panels (C4-C6). The data indicate that postinhibitory rebound was very small and thus cannot account for the slow EPSPs. Recordings were made in high-divalent

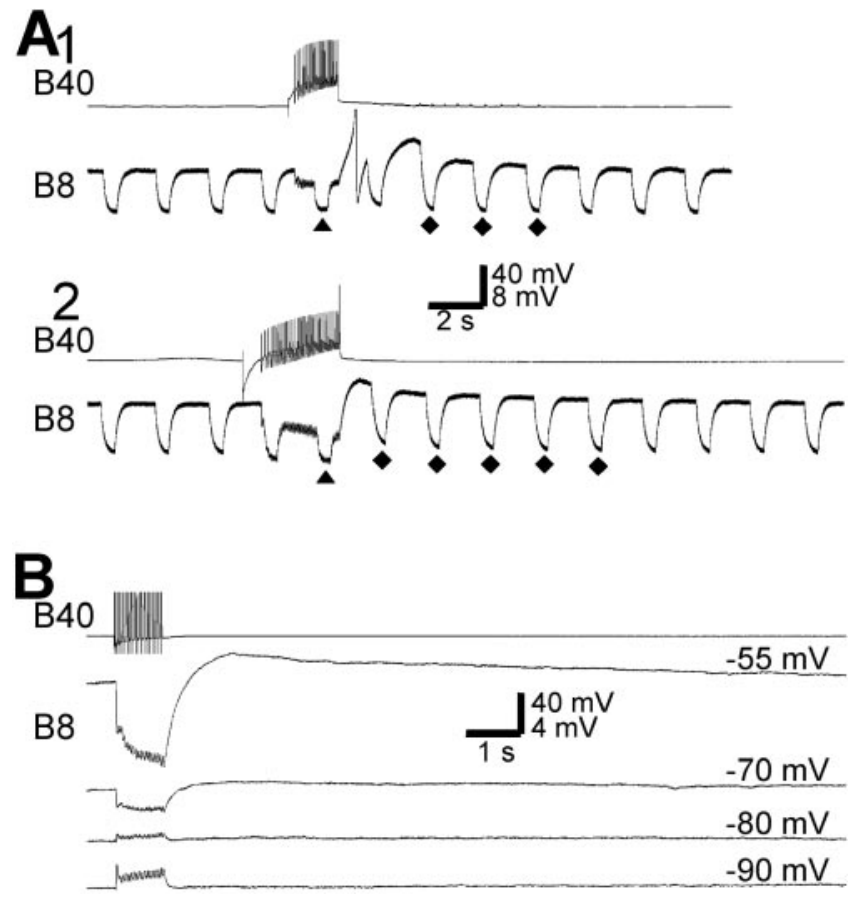

Figure 5. Synaptic potentials from B40 to the contralateral B8 are associated with conductance changes in $\mathrm{B} 8 . A, \mathrm{~B} 8$ conductance was tested as voltage deflections caused by constant hyperpolarizing current pulses of $500 \mathrm{msec}$ duration applied at $0.5 \mathrm{~Hz}$. Stimulation of B40 caused smaller voltage deflections in B8 (triangles) when the fast IPSPs were most prominent (throughout B40 stimulation), suggesting that the fast IPSPs were associated with apparent conductance increase in B8. The voltage deflections in B8 (diamonds) became larger immediately after cessation of B40 stimulation when the slow EPSPs were most prominent, thus suggesting that the slow EPSPs were associated with apparent conductance decrease in B8. Recordings were made in normal saline $(A 1)$ and highdivalent saline $(A 2)$. The single spike in $\mathrm{B} 8(A 1)$ was clipped. $B$, B40 was stimulated with brief current pulses at $20 \mathrm{~Hz}$ for $1 \mathrm{sec}$, and membrane potential of B8 (indicated at the right on top of each $B 8$ trace) was altered through current injection. The slow EPSPs became smaller when B8 was hyperpolarized and did not reverse even when B8 membrane potential was held at $-90 \mathrm{mV}$. In contrast, the fast IPSPs reversed (i.e., became depolarizing) when the membrane potential was held at and below -80 $\mathrm{mV}$. Recordings were made in high-divalent saline. Similar to the experiments shown in Figure 4, two electrodes were placed in B8: one for recording and one for current injection.

three groups (Before, B40, and After) revealed that B40 stimulation had a significant effect $\left(F_{(2,20)}=55.1 ; p<0.0001\right)$. Bonferroni multiple comparisons (post-test) of the three groups revealed that both "Before" and "After" groups are significantly different from "B40" group $(p<0.001)$, whereas Before and After groups are not significantly different from each other $(p>$ $0.05)$. For B34 effect $(n=6)$, the three groups (Before, B34, and After) did not differ from each other. The data suggest that, consistent with the data shown in Figure 3, during protraction B40 activity would reduce B8 activity while B34 activity would not. Indeed, when B40 and B34 were recorded from the same preparation, the IPSPs in B8 elicited by B34 were smaller than those elicited by B40 [compare the amplitude of hyperpolarization caused by the fast IPSPs in B8 elicited by B40 or B34

$\leftarrow$

saline. To obtain accurate measurements of membrane potentials in $\mathrm{B} 8$, all of the recordings were done with two electrodes in B8: one for recording and one for current injection. 


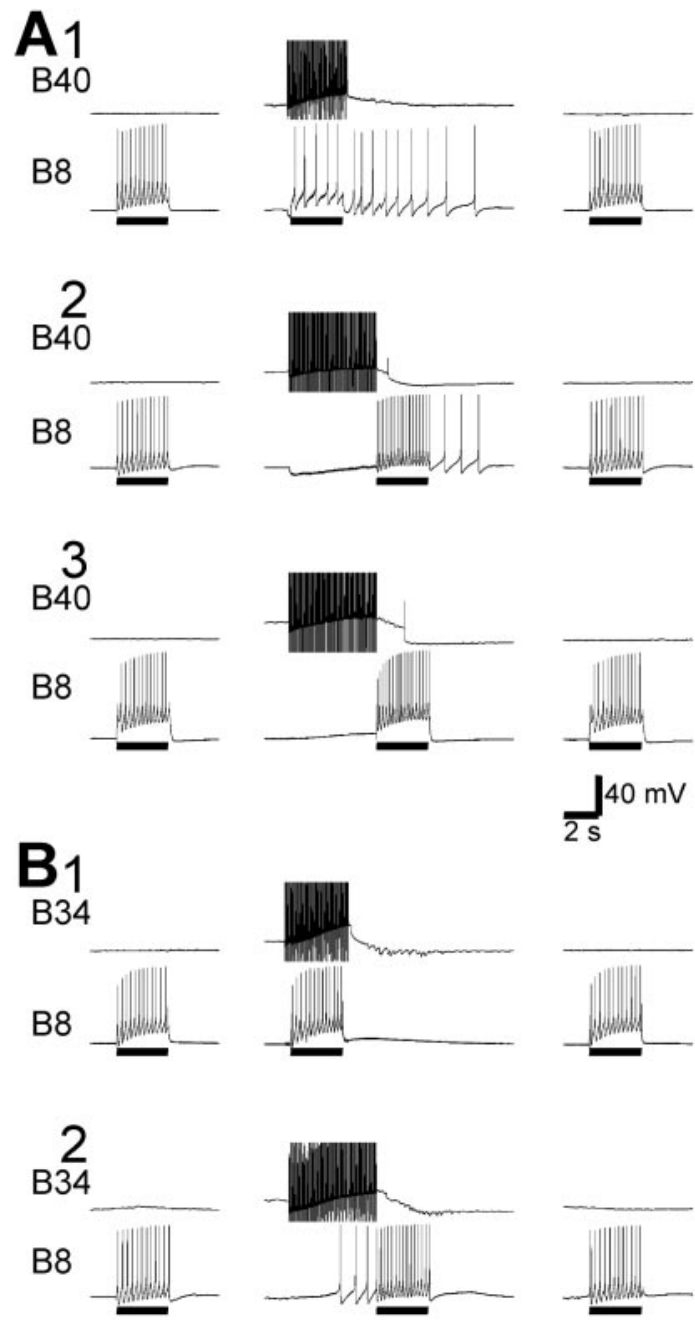

Figure 6. Effects of B40 and B34 activity on the contralateral B8 excitability. In all graphs, left panels and right panels show thethe contralateral B8 excitability, which is tested with 3 sec current pulses (bars), in control conditions of before (left) and after (right) the experimental tests. Middle panels (the experimental tests) show effects of B40 or B34 activity $(20 \mathrm{~Hz})$ on B8 excitability. In $A 1$ and B1, B40/B34 was fired $300 \mathrm{msec}$ before and throughout the current pulses (bars) injected into B8. This test paradigm examines functional effects of the fast IPSPs and simulates the situation in a feeding motor program, where B40/B34 fires together with B8 during protraction. B40 activity reduced $\mathrm{B} 8$ excitability $(A 1$, number of spikes in B8 during the $3 \mathrm{sec}$ current pulses; from left to right, 12, 5, 12 spikes), and B34 activity somewhat increased B8 excitability (B1, from left to right, 12 , 13, 12 spikes). In $A 2, A 3$, and $B 2$ (all 3 experiments were from the same preparation), B40/B34 were fired for $5 \mathrm{sec}$ immediately before the current pulses (bars) injected into B8. This test paradigm examines functional effects of the slow EPSPs and simulates the situation in an ingestive motor program, where B40/B34 fires during protraction and B8 fires during retraction, which follows protraction. B40 activity increased B8 excitability ( $A 2$, from left to right, 12, 18, 12 spikes), and B34 activity also increased $\mathrm{B} 8$ excitability ( $B 2$, from left to right $, 12,15,12$ spikes). $A 3$, To examine the potential contribution of postinhibitory rebound of $\mathrm{B} 8$ to the enhancement of B8 activity by B40 stimulation, B8 was hyperpolarized by $15 \mathrm{mV}$, and the fast response of B8 induced by B40 firing was no longer hyperpolarizing, which would eliminate postinhibitory rebound. Under these conditions, B40 activity still enhanced B8 firing (from left to right, 12, 18, 12 ), indicating that the slow excitation of B8 by B40 is indeed functional. Note that the current pulses that were used to test B8 excitability in $A 3$ were increased relative to $A 2$ so that, in the control conditions, B8 fired similar number of spikes as in $A 2$.
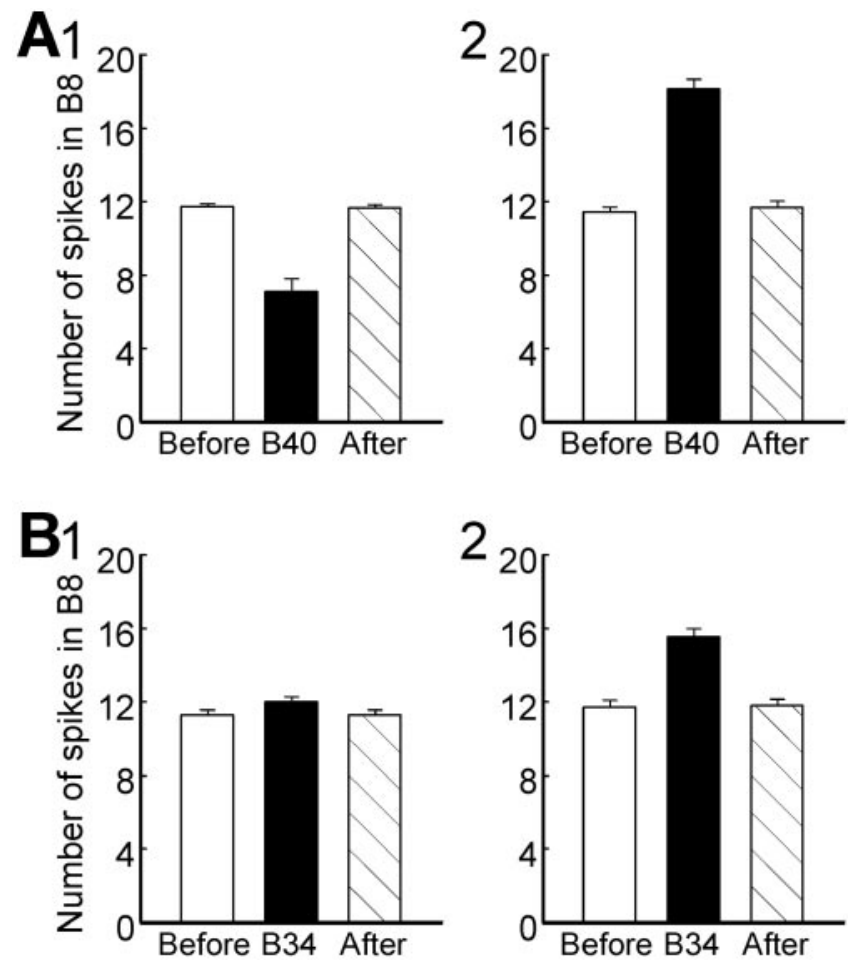

Figure 7. Plots of group data showing the effect of B40 and B34 activity on the excitability of the contralateral B8. This figure summarizes the experiments shown in Figure 6. The four bar graphs in $A 1(n=11), A 2$ $(n=9), B 1(n=6)$, and $B 2(n=6)$ correspond to examples shown in $A 1$, $A 2, B 1$, and $B 2$ in Figure 6 , respectively. When B40 or B34 was fired together with the current pulses injected into B8, B40 activity decreased B8 excitability (represented as number of spikes in B8 during the $3 \mathrm{sec}$ current pulses, $A 1)$, whereas B34 activity slightly increased it (B1), suggesting that the fast IPSPs from B40 to B8 are more effective than those from B34 to B8. When B40 or B34 was fired before the current pulses injected into $\mathrm{B} 8$, both $\mathrm{B} 40$ activity $(A 2)$ and $\mathrm{B} 34$ activity $(B 2)$ increased B8 excitability, but the increase induced by B40 was larger than that induced by B34, thus suggesting that the slow EPSPs from B40 to B8 are more effective than those from B34 to B8. See Results for statistical analysis. Error bars indicate SEM.

stimulations in the middle panels of Fig. $6 A 2$ (B40) and B2 (B34), which were recorded from the same preparation, and B8 was held at the same membrane potential].

In the second stimulation paradigm, B40 (Figs. $6 A 2, A 3,7 A 2$ ) or B34 (Figs. 6B2, 7B2) was stimulated for $5 \mathrm{sec}$ immediately before current pulses injected into B8. This paradigm examines functional effects of the slow EPSPs and simulates the situation in an ingestive motor program, where B40/B34 fire during protraction, whereas $\mathrm{B} 8$ fires during retraction. Under such conditions, both B40 (Fig. 6A2) and B34 (Fig. 6B2) activity increased B8 excitability. Group data are shown in Figure $7 A 2$ (B40), and $7 B 2$ (B34). For B40 effect $(n=9)$, one-way repeated measures ANOVA on the three groups (Before, B40, and After) revealed that B40 stimulation had a significant effect $\left(F_{(2,16)}=348.4 ; p<\right.$ 0.0001). Bonferroni multiple comparisons (post test) of the three groups revealed that both Before and After groups are significantly different from B40 group $(p<0.001)$, whereas Before and After groups are not significantly different from each other $(p>$ $0.05)$. For B34 effect $(n=6)$, one-way repeated measures ANOVA on the three groups (Before, B34, and After) also revealed that B34 stimulation had a significant effect $\left(F_{(2,12)}=\right.$ $454.8 ; p<0.0001$ ). Bonferroni multiple comparisons (post test) of 
the three groups revealed that both Before and After groups are significantly different from B34 group ( $p<0.001)$, whereas Before and After groups are not significantly different from each other $(p>0.05)$.

The increase in B8 excitability appeared larger after stimulation of B40 than stimulation of B34. To determine whether these differences are statistically significant, we performed two-way ANOVA. The two main factors in this analysis were the cell type (B40 vs B34) and stimulation conditions (Before, During, and After B40/B34 stimulation). We found that there was a significant effect of cell types $\left(F_{(1,42)}=5.76 ; p<0.05\right)$, stimulation conditions $\left(F_{(1,42)}=139.6 ; p<0.0001\right)$, and interaction of these two main factors $\left(F_{(1,42)}=11.04 ; p<0.001\right)$. Individual comparisons (post test) showed that this significant interaction was attributable to a larger increase in B8 excitability after B40 stimulation than after B34 stimulation $(p<0.001)$. There was no significant difference between the B8 excitability when neither B40 nor B34 was stimulated in two control conditions (Before, $p>0.05$; After, $p>0.05)$.

To further examine potential contribution of possible postinhibitory rebound in B8 to the enhancement of B8 firing by B40 (compare Fig. 4C4-C6) because B8 was inhibited throughout B40 firing (Figs. 4C6, 6A2), we applied a small constant hyperpolarizing current in B8 so that B40 no longer elicited fast IPSPs in B8. Under these conditions, B8 firing still increased (Fig. 6A3), suggesting that the slow EPSPs were indeed functional.

Taken together, these experiments indicate that both the fast IPSPs and the slow EPSPs from B40 to B8 are in fact functional, and B40 effects are larger than B34 effects.

\section{Synaptic connectivity of B40 within the feeding central pattern generator}

In feeding motor programs, B40 is active in protraction and inhibited in retraction. In this study, we examined the synaptic mechanism that may account for this pattern of B40 activity. One potential source of B40 excitation could be CBI-2. Indeed, CBI-2 produced facilitating EPSPs in B40 that followed presynaptic spikes one-for-one $(n=12)$ (Fig. $8 A)$. These EPSPs persisted in high-divalent saline (Fig. $8 A 2$ ), suggesting that they are monosynaptic. No reciprocal connection from B40 to CBI-2 was observed. Second source of excitation may come from B34. B34 is electrically coupled to B40 $(n=5)$ (Fig. $8 B)$. In addition, B40 is also electrically coupled to its contralateral homolog $(n=10)$ (Fig. 8B).

The inhibition may be mediated, at least partly, by the retraction-phase interneuron B64. B64 elicited IPSPs in B40 ( $n=$ 4). When B64 was stimulated with DC or current pulses, the first few B64 spikes typically did not induce visible IPSPs, but subsequent spikes elicited one-for-one IPSPs that facilitated significantly (Fig. $8 C$ ). The IPSPs also persisted in high-divalent saline (Fig. 8C2), suggesting they are monosynaptic. Thus, B64 may contribute to inhibition of B40 during the retraction phase of motor programs.

\section{DISCUSSION}

The two types of Aplysia feeding motor programs, ingestion and egestion, are generated by alterations of the relative timing of the PFMs (protraction-retraction) and the PSM (closure). Radula closure occurs in protraction in egestion, and in retraction in ingestion. The same set of interneurons (B63/B34 for protraction and B64 for retraction) fire similarly (Jing et al., 1999; Jing and Weiss, 2001) and mediate the PFMs in ingestion and egestion.
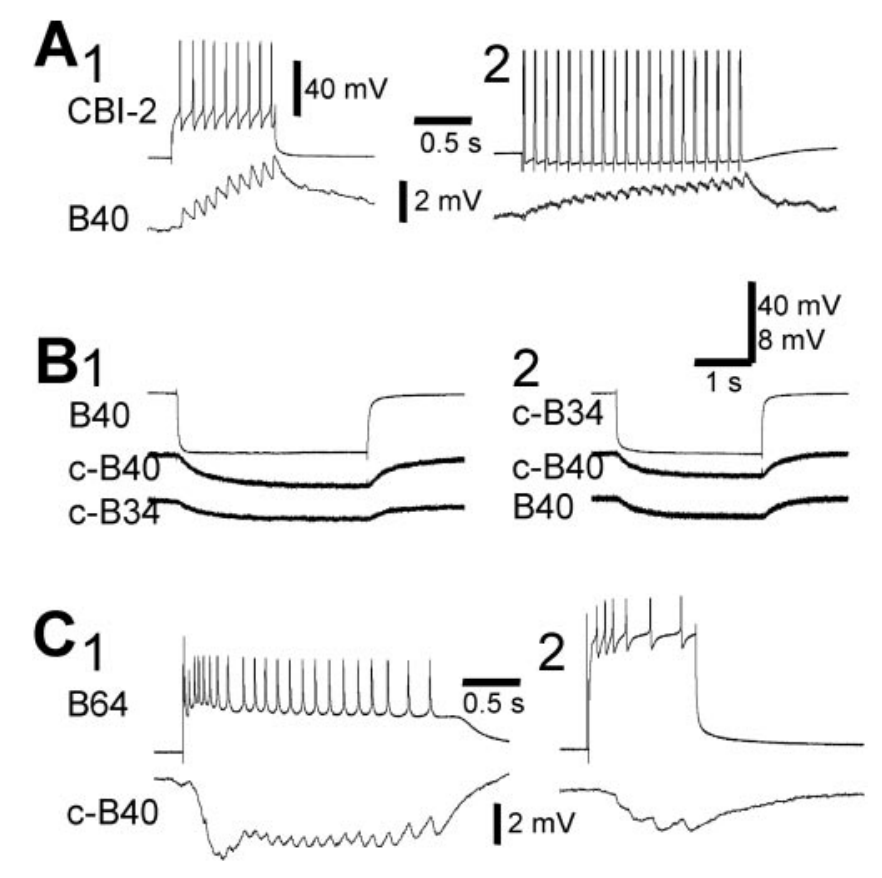

Figure 8. B40 receives excitation from protraction-phase interneurons CBI-2 and B34 and inhibition from the retraction-phase interneuron B64. $A$, CBI-2 elicited one-for-one depolarizing responses in B40 in normal saline $(A 1)$ and in high-divalent saline $(A 2)$. Recordings in $A 1$ and $A 2$ were obtained from two preparations. $B$, Negative current injection into B40 caused hyperpolarization of the contralateral B40 $(c-B 40)$ and the contralateral B34 (c-B34) (B1). Similarly, negative current injection into c-B34 caused hyperpolarization of c-B40 and ipsilateral B40 (B2). This indicates that B40 is electrically coupled to its contralateral homolog, and B34 is coupled to both the ipsilateral and contralateral B40. Recordings were obtained from the same preparation in high-divalent saline. $C, \mathrm{~B} 64$ elicited IPSPs in the contralateral B40 (c-B40) that followed presynaptic spikes one-for-one in normal saline $(C 1)$ and high-divalent saline $(C 2)$. Recordings were obtained from the same preparation.

This notion is reinforced by our finding that B34, which excites strongly protraction motorneurons (Hurwitz et al., 1997), has a small impact on the activity of B8, a closure motorneuron (Fig. 3). We have shown that B8 phasing in egestion is mediated by B20, which is preferentially active in egestion, is active in protraction, and uses fast excitation to drive B8 (Jing and Weiss, 2001). Here, we identified an interneuron B40 that is preferentially active in ingestion and is crucial for the expression of ingestive programs (Fig. 9C).

Unexpectedly, B40 is active in protraction, but not active in retraction, the phase in which $\mathrm{B} 8$ is most active in ingestion. Consequently, B40 cannot use fast excitation to drive B8 in retraction as B20 does in protraction. Instead, B40 uses dual component PSPs, fast inhibition and slow excitation to promote B8 activity in retraction. In functional terms, the delayed excitation of B8 by B40 activity during protraction is equivalent to the fast excitation that B40 would have to exert if it were active in retraction. One implication of these findings is that activity phasing of interneurons may actually be different from the activity phasing of the motor neurons they control. Thus, caution should be exercised when interpreting data based solely on the phase of activity of interneurons.

While the functional role of the slow excitation from B40 to B8 is to drive closure during retraction in ingestive programs, the functional role of the fast inhibition from B40 to B8 is less obvious. This may be understood from two perspectives. First, the 

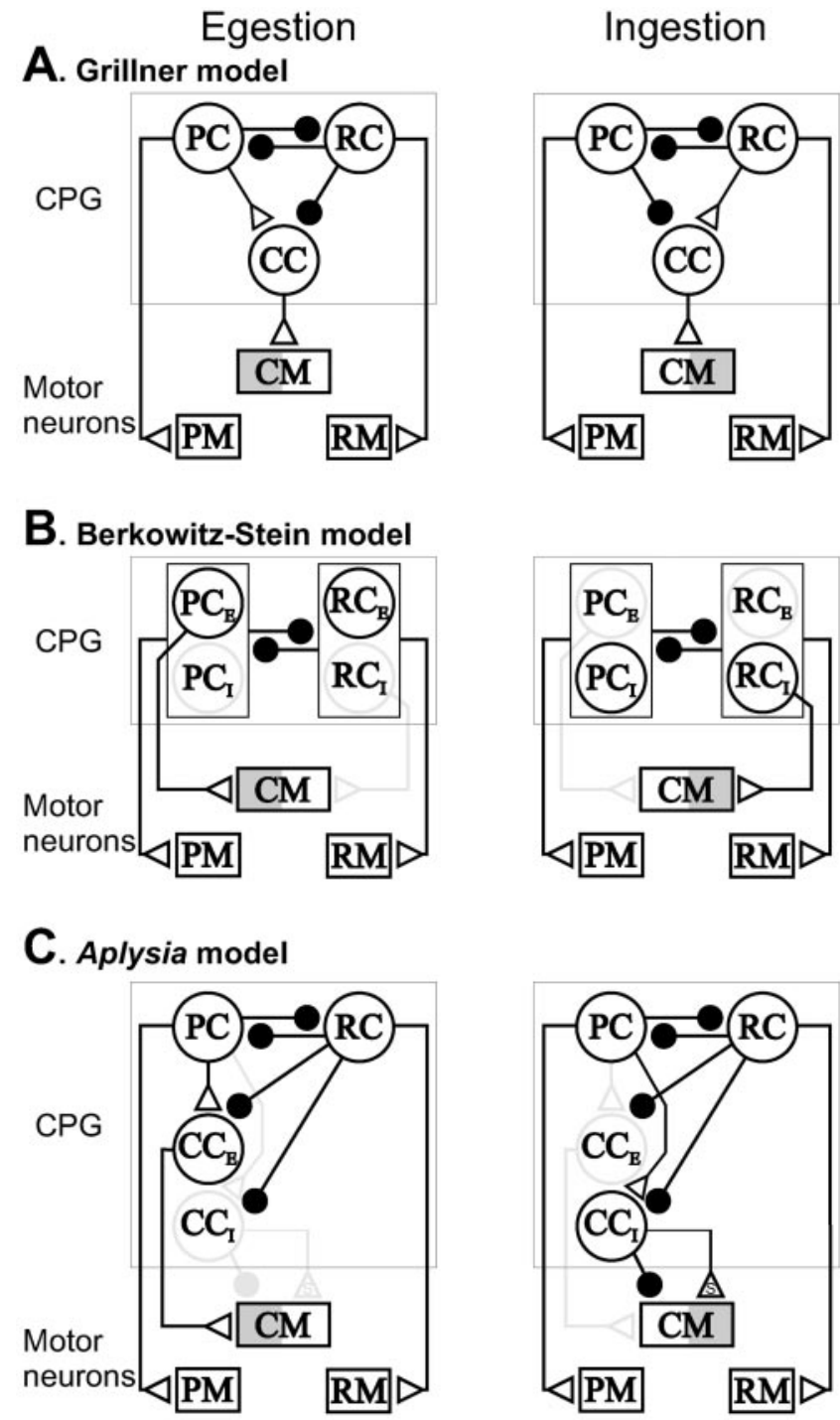

Cell name translation for $C$ :
$\begin{array}{ll}\mathrm{PC}=\mathrm{B} 63, \mathrm{~B} 34 & \mathrm{RC}=\mathrm{B} 64 \\ \mathrm{CC}_{\mathrm{E}}=\mathrm{B} 20 & \mathrm{CC}_{1}=\mathrm{B} 40 \\ \mathrm{PM}=\mathrm{B} 31 / 32, \mathrm{~B} 61 / 62 & \mathrm{CM}=\mathrm{B} 8\end{array}$

Figure 9. Alternative network models of the generation of egestive and ingestive motor programs in Aplysia feeding. The two motor programs require coordination of two sets of radula movements, protractionretraction and opening-closing. Each rhythmic cycle consists of alternating protraction and retraction phases that are represented by protraction motor neurons $(P M)$ and retraction motor neurons $(R M)$, respectively, in the diagrams. In each cycle, protraction precedes retraction, whereas the radula closure shifts its phase relative to protraction-retraction. Thus, in egestive motor programs (left panels), the radula closure motor neuron (CM, B8) is active in protraction (depicted as gray shades in the left half of $\mathrm{CM}$ ), whereas in ingestive motor programs (right panels), $\mathrm{CM}$ is active in retraction (depicted as gray shades in the right half of $\mathrm{CM}$ ). The phasing of motorneuronal activity is mediated by interneurons of a central pattern generator $(C P G)$. PM and RM are controlled by protraction controller interneurons (PC, B63, and B34) and retraction controller interneuron (RC) (e.g., B64), respectively. PC and $\mathrm{RC}$ reciprocally inhibit each other (Hurwitz et al., 1997), thus forming a half-center oscillator. A, A model based on the Grillner (1981, 1985) hypothesis in spinal circuit (Grillner, 1981, 1985). The main feature of the model is that interneurons (i.e., controllers) for radula movements [PC, RC, as well as closure controller interneurons $(C C)]$ are shared between ingestion and egestion. Phase shifting of CM between protraction and retraction in the two programs is mediated by phase shifting of CC through a change of sign of coupling that is implemented by descending fibers. Specifically, in egestion, CC onset of B8 activation should occur at the beginning of retraction, not earlier, but the slow excitation that B40 uses to drive B8 cannot achieve precise timing on its own. The fast inhibition can thus be used to ensure that B8 is activated to fire at high rates only during retraction when B40 halts its firing and when the slow excitation is still effective but the fast inhibition ceases to exist. Second, one essential feature of the circuit is that changes in the activity phase of radula closure are implemented by recruitment of separate interneurons B20 and B40 (Fig. 9). Both interneurons are active during protraction and receive excitation from partially overlapping sets of protraction-phase interneurons, e.g., CBI-2 and B34, that lead to a certain degree of coactivation of B20 and B40 in some motor programs (Fig. 1). Because activity of B20 in ingestive programs can generate a dysfunctional increase in B8 firing during protraction, B40-elicited fast inhibition can thus counter the effects of B20 activity. This notion is supported by observations that hyperpolarization of B40 resulted not only in the decrease of B8 firing in retraction but also in its increase during protraction. Conversely, in egestive programs, alternate mechanisms must be present to prevent B8 activity in retraction that may be promoted by coactivation of B40 with B20. This function is accomplished by a separate element B4/5, which B20 excites (Jing and Weiss, 2001). In ingestion, the dual-component PSPs from B40 accomplishes two functions. Such implementation is thus effective and efficient.

One important feature of the Aplysia circuit (Fig. 9C) is that activity of interneurons for the PSM is coordinated with those for the PFMs through direct synaptic connections between these interneurons. The interneurons for the PFMs, B63, B34 (protraction) and B64 (retraction) reciprocally inhibit each other (Hurwitz et al., 1997), thus forming a half-center oscillator. Interneurons $\mathrm{B} 20$ and $\mathrm{B} 40$ for the PSM receive excitation from protraction-phase interneurons (e.g., CBI-2, B34) and are inhibited by retraction-phase interneuron (B64). Thus, they are active

is driven by $\mathrm{PC}$ and inhibited by $\mathrm{RC}$, whereas in ingestion, $\mathrm{CC}$ is driven by RC and inhibited by PC. B, A model based on the Berkowitz and Stein (1994) model of the turtle-scratching spinal circuit. In this model, protraction and retraction are mediated by partially overlapping sets of PC and RC interneurons. In egestion, PCs, which are maximally tuned to egestion $\left(P C_{E}\right)$, excite both $\mathrm{PM}$ and $\mathrm{CM}$, thus $\mathrm{CM}$ is active together with $\mathrm{PM}$ Similarly in ingestion, $\mathrm{RCs}$, which are maximally tuned to ingestion $\left(\mathrm{RC}_{\mathrm{I}}\right)$, excite both RM and CM, thus B8 is active together with RM. $C$, The Aplysia feeding network model that is based on present and earlier studies on feeding interneurons (Hurwitz and Susswein, 1996; Hurwitz et al., 1997; Jing et al., 1999; Jing and Weiss, 2001). Translation of cell names that are mentioned in the paper to functional terms $\left(P C, R C, C C_{E}, C C_{I}, P M, C M\right)$ is provided at the bottom. Similar to the Grillner $(1981,1985)$ model, PC and $\mathrm{RC}$ remain the same in both egestion and ingestion. On the other hand, similar to the Berkowitz and Stein (1994) model, two separate CCs $\left(C_{\mathrm{E}}\right.$ for egestion and $\mathrm{CC}_{\mathrm{I}}$ for ingestion) are recruited to mediate $\mathrm{CM}$ phasing in the two motor programs. In egestion, $\mathrm{CC}_{\mathrm{E}}(\mathrm{B} 20)$ receives excitation from $\mathrm{PC}$ and inhibition from $\mathrm{RC}$, so it is active in protraction. $\mathrm{CC}_{\mathrm{E}}$ drives $\mathrm{CM}$ directly through fast excitation so $\mathrm{CM}$ is active in protraction. In addition, a separate neuron $\mathrm{B} 4 / 5$, which is preferentially active in egestion and active in retraction, inhibits B8 to prevent B8 firing in retraction (Jing and Weiss, 2001) (not depicted for clarity). In ingestion, $C_{C_{I}}$ (B40) also receives excitation from $\mathrm{PC}$ and inhibition from $\mathrm{RC}$, so it is active in protraction. However, unlike $\mathrm{CC}_{\mathrm{E}}, \mathrm{CC}_{\mathrm{I}}$ does not use fast excitation; instead, it uses slow excitation to promote $\mathrm{CM}$ activity in retraction. In addition, $\mathrm{CC}_{\mathrm{I}}$ exerts fast inhibition on $\mathrm{CM}$ to precisely activate $\mathrm{CM}$ at the onset of retraction and prevent excessive $\mathrm{B} 8$ activity in protraction that may be promoted by $\mathrm{CC}_{\mathrm{E}}$ (see Discussion). An $s$ within the connection symbols $(C)$ depicts "slow synaptic connections." Functionally ineffective synaptic connections and weakly active neurons are shown in gray. 
only in protraction and they, together with interneurons for the PFMs, form a single, multifunctional, central pattern generator (CPG) (Delcomyn, 1980; Getting, 1989; Marder and Calabrese, 1996). This coordination scheme contrasts with intersegmental coordination between multiple segmental oscillators in lamprey (Cohen et al., 1992; Williams, 1992; Grillner et al., 1995), leech (Friesen and Pearce, 1993), and crayfish (Tschuluun et al., 2001). In these latter cases, the timing of sets of movements in each segment is determined by activity of each oscillator, and the timing of overall behaviors has to be coordinated through more elaborate coupling mechanisms between these oscillators.

\section{Comparison with other models of movement coordination}

Two models of spinal circuits were proposed to explain intralimb coordination in forward-backward locomotion (Grillner, 1981, 1985) and in turtle rostral and pocket scratch (Berkowitz and Stein, 1994). For comparison, we translated these two spinal models into the Aplysia feeding circuit (Fig. 9).

One major stipulation of the Grillner $(1981,1985)$ model is that a unit burst generator, or a controller (Orlovsky et al., 1999), exists for each agonist muscle movement. In terms of movement control, we define a controller as an interneuron or interneurons that provides direct excitation to a relevant motorneuron or motorneurons and whose activity is important, in a motor program-specific manner, to determine the activity of the motorneuron or motorneurons. Aplysia version of the Grillner (1981, 1985) model postulates separate controllers for protraction (PC), retraction (RC), and closure (CC). Aplysia version of the Berkowitz and Stein (1994) model postulates no separate controllers for closure. Instead, closure is controlled directly by protraction controllers $\left(\mathrm{PC}_{\mathrm{E}}\right)$ that are preferentially active in egestion or by retraction controllers $\left(\mathrm{RC}_{\mathrm{I}}\right)$ that are preferentially active in ingestion. Aplysia studies support the existence of separate controllers for different movements and also different $\mathrm{CC}$ s for egestion $\left(\mathrm{CC}_{\mathrm{E}}\right)$ and ingestion $\left(\mathrm{CC}_{\mathrm{I}}\right)$ (see the beginning of Discussion). However, the distinction between the controllers is not as strict as in the Grillner $(1981,1985)$ model, because B34, as a PC, does have some effect on B8 activity, albeit small. Thus, our model incorporates features of both spinal models, because the interneurons for PFMs remain the same as is the case in the Grillner (1981, 1985) model, but preferential activation of different interneurons implements the PSM in ingestion and egestion as is the case in the Berkowitz and Stein (1994) model.

Organizational differences of movement controllers in the three models have several implications for the mechanisms that generate different motor programs. In the Grillner (1981, 1985) model, CC does not change in different programs, and descending fibers change the sign of coupling between $\mathrm{PC} / \mathrm{RC}$ and $\mathrm{CC}$ so that $\mathrm{CC}$ changes the phase together with closure motorneurons. In contrast, both in the Berkowitz and Stein (1994) and our models, $\mathrm{CC}$ does not change its activity phase, instead, separate CCs are recruited into different programs. Thus, these two models are similar and can be classified as recruitment model. Specifically, in both models, closure motorneurons are controlled by two separate sets of interneurons in the two programs. These two CCs are active in both programs, but display different activity levels. This scheme is consistent with population coding that has been described in behavioral choice and directional behaviors (Kristan and Shaw, 1997; Sparks et al., 1997), such as leech bending (Lockery and Kristan, 1990) and cockroach escape turns (Levi and Camhi, 2000). To generate different motor programs, sensory and/or descending fibers or other mechanisms must exist to preferentially activate $\mathrm{PC}_{\mathrm{E}}$ for the Berkowitz and Stein (1994) model, or $\mathrm{CC}_{\mathrm{E}}$ for our model in egestion. The same scenario applies for $\mathrm{RC}_{\mathrm{I}}$ or $\mathrm{CC}_{\mathrm{I}}$ for ingestion. However, the Berkowitz and Stein (1994) and our models differ in how closure is controlled in ingestion. Closure is implemented by dual excitation of two motor pools in the former model, and by a novel mechanism, fast-inhibition and slow-excitation, in the latter model.

In addition to Aplysia feeding (Morton and Chiel, 1993a) and turtle-scratching (Mortin et al., 1985), there are several examples in which related motor programs are generated by different combinations of multiple sets of relevant movements, e.g., different forms of locomotion in cat (Perret and Cabelguen, 1980; Smith et al., 1985, 1998a; Buford and Smith, 1990; Pearson and Rossignol, 1991; Rossignol, 1996; Carlson-Kuhta et al., 1998), crayfish (Ayers and Davis, 1977; Chrachri and Clarac, 1990), and locust (Duch and Pfluger, 1995), feeding in mollusks (Croll and Davis, 1981) and lobster (Combes et al., 1999). Although it is interesting to compare the mechanisms in Aplysia with other systems, there are few detailed circuitry or mechanistic-level studies available to make meaningful comparisons. One exception may be the gastric mill system of lobster stomatogastric ganglion, in which the motor sequences of two sets of teeth movements in type I and type II motor programs are similar to those of radula movements in egestive and ingestive motor programs in Aplysia (Combes et al., 1999). However, neural organization of the stomatogastric ganglion is simpler than that of Aplysia in that the motor neurons are by themselves CPG elements (see Discussion in Jing and Weiss, 2001). Thus, the change of the phasing of the PSMs relative to the PFMs are implemented by the direct change of phasing of relevant motor neurons, rather than being implemented by a separate layer of CPG interneurons as is the case in Aplysia (Fig. 9) and vertebrate spinal circuits.

In summary, by elucidating specific roles of Aplysia feeding interneurons involved in generation of two coordinated motor programs, we established a neural model which has some similarities to but also difference from the two spinal models for vertebrate intralimb coordination (Grillner, 1981, 1985; Berkowitz and Stein, 1994), and thus the significance of our findings may extend beyond the Aplysia feeding network.

\section{REFERENCES}

Ayers Jr JL, Davis WJ (1977) Neuronal control of locomotion in the lobster, Homarus americanus. I. motor programs for forward and backward walking. J Comp Physiol [A] 115:1-28.

Berkowitz A, Stein PS (1994) Activity of descending propriospinal axons in the turtle hindlimb enlargement during two forms of fictive scratching: phase analyses. J Neurosci 14:5105-5119.

Buford JA, Smith JL (1990) Adaptive control for backward quadrupedal walking. II. Hindlimb muscle synergies. J Neurophysiol 64:756-766.

Buford JA, Zernicke RF, Smith JL (1990) Adaptive control for backward quadrupedal walking. I. Posture and hindlimb kinematics. J Neurophysiol 64:745-755.

Carlson-Kuhta P, Trank TV, Smith JL (1998) Forms of forward quadrupedal locomotion. II. A comparison of posture, hindlimb kinematics, and motor patterns for upslope and level walking. J Neurophysiol 79:1687-1701.

Chiel HJ, Weiss KR, Kupfermann I (1986) An identified histaminergic neuron modulates feeding motor circuitry in Aplysia. J Neurosci 6:2427-2450.

Chrachri A, Clarac F (1990) Fictive locomotion in the fourth thoracic ganglion of the crayfish, Procambarus clarkii. J Neurosci 10:707-719.

Church PJ, Lloyd PE (1994) Activity of multiple identified motor neurons recorded intracellularly during evoked feedinglike motor programs in Aplysia. J Neurophysiol 72:1794-1809.

Cohen AH, Ermentrout GB, Kiemel T, Kopell N, Sigvardt KA, Williams TL (1992) Modelling of intersegmental coordination in the lamprey central pattern generator for locomotion. Trends Neurosci 15:434-438. Combes D, Meyrand P, Simmers J (1999) Motor pattern specification by 
dual descending pathways to a lobster rhythm-generating network. J Neurosci 19:3610-3619.

Croll RP, Davis WJ (1981) Motor program switching in Pleurobranchaea: I. Behavioral and electromyographic study of ingestion and egestion in intact specimens. J Comp Physiol 145:277-287.

Cropper EC, Kupfermann I, Weiss KR (1990) Differential firing patterns of the peptide-containing cholinergic motor neurons B15 and B16 during feeding behavior in Aplysia. Brain Res 522:176-179.

Delcomyn F (1980) Neural basis of rhythmic behavior in animals. Science 210:492-498.

Duch C, Pfluger HJ (1995) Motor patterns for horizontal and upside down walking and vertical climbing in the locust. J Exp Biol 198:1963-1976.

Friesen WO, Pearce RA (1993) Mechanisms of intersegemental coordination in leech locomotion. Semin Neurosci 5:41-47.

Getting PA (1989) Emerging principles governing the operation of neural networks. Annu Rev Neurosci 12:185-204.

Grasso R, Bianchi L, Lacquaniti F (1998) Motor patterns for human gait: backward versus forward locomotion. J Neurophysiol 80:1868-1885.

Grillner S (1981) Control of locomotion in bipeds, tetrapods, and fish. In: Handbook of Physiology, Sec 1, Vol 2 (Brooks V, ed), pp 1179-1236. Bethesda, MD: American Physiological Society.

Grillner S (1985) Neurobiological bases of rhythmic motor acts in vertebrates. Science 228:143-149.

Grillner S, Deliagina TG, Ekeberg O, El Manira A, Hill RH, Lansner A, Orlovsky GN, Wallen P (1995) Neural networks that co-ordinate locomotion and body orientation in lamprey. Trends Neurosci 18:270-279.

Hurwitz I, Susswein AJ (1996) B64, a newly identified central pattern generator element producing a phase switch from protraction to retraction in buccal motor programs of Aplysia californica. J Neurophysiol 75:1327-1344.

Hurwitz I, Neustadter D, Morton DW, Chiel HJ, Susswein AJ (1996) Activity patterns of the B31/B32 pattern initiators innervating the I2 muscle of the buccal mass during normal feeding movements in Aplysia californica. J Neurophysiol 75:1309-1326.

Hurwitz I, Kupfermann I, Susswein AJ (1997) Different roles of neurons B63 and B34 that are active during the protraction phase of buccal motor programs in Aplysia californica. J Neurophysiol 78:1305-1319.

Jing J, Weiss KR (2001) Neural mechanisms of motor program switching in Aplysia. J Neurosci 21:7349-7362.

Jing J, Morgan PT, Hurwitz I, Cropper EC, Weiss KR (1999) Differential roles of buccal elements of the feeding central pattern generator (CPG) in switching CBI-2-induced motor programs in Aplysia. Soc Neurosci Abstr 25:1643.

Jordan LM (1991) Brainstem and spinal cord mechanisms for the initiation of locomotion: single limb analysis. In: Neurobiological basis of human locomotion (Shimamura M, Grillner S, Edgerton VR, eds), pp 3-20. Tokyo: Japan Scientific Societies.

Kabotyanski EA, Baxter DA, Byrne JH (1998) Identification and characterization of catecholaminergic neuron B65, which initiates and modifies patterned activity in the buccal ganglia of Aplysia. J Neurophysiol 79:605-621.

Kristan Jr WB, Shaw BK (1997) Population coding and behavioral choice. Curr Opin Neurobiol 7:826-831.

Kupfermann I (1974) Feeding behavior in Aplysia: a simple system for the study of motivation. Behav Biol 10:1-26.

Levi R, Camhi JM (2000) Wind direction coding in the cockroach escape response: winner does not take all. J Neurosci 20:3814-3821.

Lockery SR, Kristan WB Jr (1990) Distributed processing of sensory information in the leech. II. Identification of interneurons contributing to the local bending reflex. J Neurosci 10:1816-1829.

Marder E, Calabrese RL (1996) Principles of rhythmic motor pattern generation. Physiol Rev 76:687-717.

Morgan PT, Jing J, Vilim FS, Weiss KR (2002) Interneuronal and pep- tidergic control of motor pattern switching in Aplysia. J Neurophysiol 87:49-61.

Mortin LI, Keifer J, Stein PS (1985) Three forms of the scratch reflex in the spinal turtle: movement analyses. J Neurophysiol 53:1501-1516.

Morton DW, Chiel HJ (1993a) In vivo buccal nerve activity that distinguishes ingestion from rejection can be used to predict behavioral transitions in Aplysia. J Comp Physiol 172:17-32.

Morton DW, Chiel HJ (1993b) The timing of activity in motor neurons that produce radula movements distinguishes ingestion from rejection in Aplysia. J Comp Physiol 173:519-536.

Nargeot R, Baxter DA, Byrne JH (1997) Contingent-dependent enhancement of rhythmic motor patterns: an in vitro analog of operant conditioning. J Neurosci 17:8093-8105.

Orlovsky GN, Deliagina TG, Grillner S (1999) Neural control of locomotion. New York: Oxford UP.

Pearson KG, Rossignol S (1991) Fictive motor patterns in chronic spinal cats. J Neurophysiol 66:1874-1887.

Perret C, Cabelguen JM (1980) Main characteristics of the hindlimb locomotor cycle in the decorticate cat with special reference to bifunctional muscles. Brain Res 187:333-352.

Robertson GA, Mortin LI, Keifer J, Stein PS (1985) Three forms of the scratch reflex in the spinal turtle: central generation of motor patterns. J Neurophysiol 53:1517-1534.

Rosen SC, Teyke T, Miller MW, Weiss KR, Kupfermann I (1991) Identification and characterization of cerebral-to-buccal interneurons implicated in the control of motor programs associated with feeding in Aplysia. J Neurosci 11:3630-3655.

Rossignol S (1996) Neural control of stereotypic limb movements. In: Handbook of physiology (Rowell LB, Sheperd JT, eds), pp 173-216. New York: Oxford UP.

Sanchez JA, Kirk MD (2000) Short-term synaptic enhancement modulates ingestion motor programs of Aplysia. J Neurosci 20:RC 85:1-7.

Smith JL, Hoy MG, Koshland GF, Phillips DM, Zernicke RF (1985) Intralimb coordination of the paw-shake response: a novel mixed synergy. J Neurophysiol 54:1271-1281.

Smith JL, Carlson-Kuhta P, Trank TV (1998a) Forms of forward quadrupedal locomotion. III. A comparison of posture, hindlimb kinematics, and motor patterns for downslope and level walking. J Neurophysiol 79:1702-1716.

Smith JL, Carlson-Kuhta P, Trank TV (1998b) Motor patterns for different forms of walking: cues for the locomotor central pattern generator. Ann NY Acad Sci 860:452-455.

Sparks DL, Kristan WBJ, Shaw BK (1997) The role of population coding in the control of movement. In: Neurons, networks, and motor behavior (Stein PS, Grillner S, Selverston AI, Stuart DG, eds), pp 21-32. Cambridge, MA: MIT.

Stein PSG, Smith JL (1997) Neural and biomechanical control strategies for different forms of vertebrate hindlimb motor tasks. In: Neurons, networks, and motor behavior (Stein PSG, Grillner S, Selverston AI, Stuart DG, eds), pp 61-73. Cambridge, MA: MIT.

Stein PS, Victor JC, Field EC, Currie SN (1995) Bilateral control of hindlimb scratching in the spinal turtle: contralateral spinal circuitry contributes to the normal ipsilateral motor pattern of fictive rostral scratching. J Neurosci 15:4343-4355.

Susswein AJ, Byrne JH (1988) Identification and characterization of neurons initiating patterned neural activity in the buccal ganglia of Aplysia. J Neurosci 8:2049-2061.

Tschuluun N, Hall WM, Mulloney B (2001) Limb movements during locomotion: tests of a model of an intersegmental coordinating circuit. J Neurosci 21:7859-7869.

Weiss KR, Shapiro E, Kupfermann I (1986) Modulatory synaptic actions of an identified histaminergic neuron on the serotonergic metacerebral cell of Aplysia. J Neurosci 6:2393-2402.

Williams TL (1992) Phase coupling by synaptic spread in chains of coupled neuronal oscillators. Science 258:662-665. 